\title{
Maximum Takeoff Gross Weight of Aircraft in Search and Rescue Sorties
}

\author{
Mohd Harridon Mohamed Suffian, Mohamad Dali Isa, Hazariah Mohd Noh, Nurhayati Mohd Nur
}

\begin{abstract}
Several aircraft types are commonly used to perform many search and rescue missions throughout the world. Aircraft have been used because they are able to offer a bird's eye view of the surrounding search areas. In general, these aircraft come in various shapes, sizes and weights, and have myriad capabilities in carrying rescued and/or injured personnel and also equipment for the search and medical operations during the search and rescue sorties. For an aircraft, takeoff gross weight is important because it will affect the flying performance of the aircraft. To perform an effective search and rescue mission, the right selection of aircraft is essential such that unnecessary resources are not being wasted. In line with this notion, this study examines the distribution of the maximum takeoff gross weight of aircraft that have been typically used in search and rescue sorties. This knowledge will help in the design of future aircraft for such missions and also narrowing the selection of existing aircraft to be used on that kind of operations. It is found from the data analysis that most current operators have been using medium-haul aircraft that have a takeoff gross weight between 4,301 $\mathrm{kg}$ and $8,600 \mathrm{~kg}$.
\end{abstract}

Keywords: search and rescue, takeoff gross weight, aircraft, performance analysis.

\section{INTRODUCTION}

Numerous aircraft have been used for search and rescue operations worldwide. These aircraft are typically deployed at various locations and terrains, and carry specific equipment or different numbers of rescued victims. The environment is that the aircraft is flying can also be at mixed levels between easy and hard. With such operating conditions, it is imperative for the operator to choose an appropriate and suitable aircraft for performing the search and rescue sorties. This is to ensure that available resources are optimized in order not to waste them while still maintaining operational effectiveness level for the search and rescue mission. For instance, it is rather wasteful to fly an aircraft that has a range of $7,000 \mathrm{~km}$ when it has already

Revised Manuscript Received on December 30, 2019.

* Correspondence Author

Mohd Harridon Mohamed Suffian*, Universiti Kuala Lumpur Malaysian Institute of Aviation Technology, Dengkil, Selangor, Malaysia. E-mail: mdharridon@unikl.edu.my

Mohamad Dali Isa, Universiti Kuala Lumpur Malaysian Institute of Aviation Technology, Dengkil, Selangor, Malaysia.

Hazariah Mohd Noh, Universiti Kuala Lumpur Malaysian Institute of Aviation Technology, Dengkil, Selangor, Malaysia.

Nurhayati Mohd Nur, Universiti Kuala Lumpur Malaysian Institute of Aviation Technology, Dengkil, Selangor, Malaysia.

(C) The Authors. Published by Blue Eyes Intelligence Engineering and Sciences Publication (BEIESP). This is an open access article under the CC BY-NC-ND license (http://creativecommons.org/licenses/by-nc-nd/4.0/) confirmed that the search area is small. In similar manner, it is also wasteful to operate a huge aircraft with an inherently high maximum takeoff gross weight (MTOW) in a rescue mission for just one individual.

Among the aircraft that have been commonly operated for the search and rescue mission worldwide is the AW149. It is a helicopter that is used by various parties throughout the world such as Thailand to perform the search and rescue operations. With a MTOW of 8,600 kg, the AW149 can be equipped with ground attack armament that makes it suitable to be operated for combat rescue missions in hostile environment where the armament can be used as a defensive mechanism during such operations [1]. The AW149 helicopter is able to carry up to 18 passengers. However, in most search and rescue applications, the aircraft's cabin configuration is often changed or modified to accommodate medical equipment and proper space for the transport of injured personnel, which will effectively reduce the maximum number of passengers that it can carry onboard. On the other hand, another typical aircraft that have been used in many search and rescue missions worldwide is the Dornier Do 228. This utility aircraft, which is of twin-turboprop type, has been used by many countries including Germany, Finland, Netherlands and several others to conduct ocean patrols and also search and rescue operations. Bearing those applications in mind, the majority of this aircraft's weight can be attributed to the weights of search equipment and radar to facilitate the visual observation in the operations, and its MTOW totals up to $5,700 \mathrm{~kg}$ [2]. The Dornier Do 228 aircraft is often operated for long range search missions, which means it can cover a big search area. Furthermore, the V-22 Osprey aircraft is capable of rescuing troops in the battlefield in a swift manner since its design is essentially a hybrid between fixed wing aircraft and helicopter. It is being operated by the United States military and even though it has a bigger built compared to most rescue helicopter designs (and therefore the capability of rescuing a maximum of 22 troops), it still offers a much faster action than the others [3]. Its total MTOW of $27,400 \mathrm{~kg}$ is considered as very heavy but this is countered by its capability to hold more people and be equipped with state-of-the-art weapon systems that have a hefty weight, making the V-22 Osprey aircraft a formidable choice by the United States military [4]. Last but not least, another aircraft type that has been operated in search and rescue missions is Sikorsky S-70 Firehawk /Blackhawk. It is a multiple-role helicopter, which is capable to be used for troops insertion in a battlefield and also for combat search and rescue. 
With its rescue hoist and interior gun, it has the ability to perform rescue operations in an optimum manner [5]. Its cabin volume allows it to be fitted with medical equipment for conducting pre-surgery procedures. The MTOW for Sikorsky S-70 Firehawk/Blackhawk helicopter is 10,000 kg. Since takeoff gross weight is one of the important aircraft design parameters that can affect its performance, it should be of high consideration when designing new aircraft or selecting appropriate aircraft for a particular mission. In this study, the focus is placed on aircraft for the search and rescue operations and their takeoff gross weight. It is crucial to operate the right aircraft for the mission to have a highly effective mission and reduce any waste of resources.

\section{METHODOLOGY}

Overall methodology that has been followed for this study is presented in Fig. 1. In short, 40 aircraft that have been used in most search and rescue operations worldwide are identified and studied. Once these aircraft have been recognized, their MTOW data is collected and then statistically analyzed using scatter plots. The identified outliers in the plot are removed in order to give an accurate description of the collected MTOW data. As mentioned in [6], existence of outliers can decrease the accuracy of the data representation and by removing them, it will enhance the fit of the constructed mathematical model and/or better reflect the true meaning of the data set. This has been demonstrated in [7], where pruning process to eradicate outliers is done and the resultant database has become more acceptable in terms of unearthing underlying pattern or trend of the data. In the study, removal of outliers in an incremental fashion is shown to be effective in gaining higher confidence for the data set. Meanwhile, another study in [8] highlights the importance of removing outliers to increase accuracy of the analysis for the data set. For this study, outliers are identified using the graphical mean, which is an acceptable approach as outlined in Minitab software [11]. With the outliers removed, a new set of data that is deemed more congruent is obtained. The resultant new average line is constructed and the MTOW data is then analyzed accordingly.

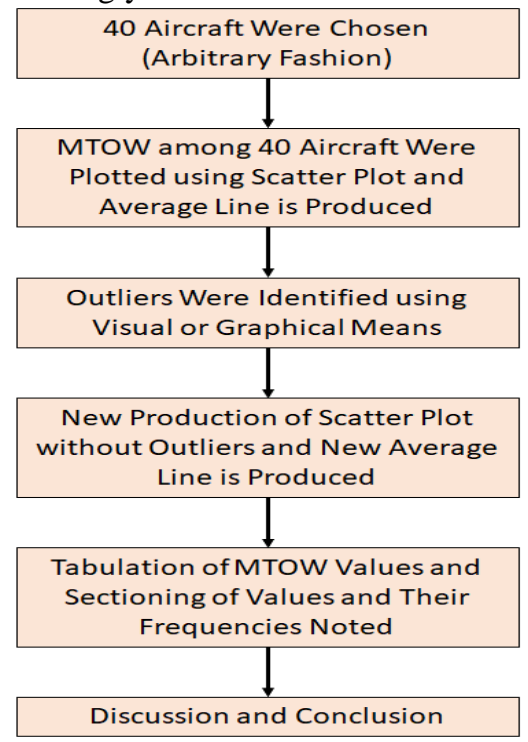

Fig.1. Methodology framework of conducted study

\section{RESULTS AND DISCUSSION}

Fig. 2 presents the plot of maximum takeoff gross weight of the typical aircraft used for search and rescue missions. It can be noted that the majority of the aircraft has maximum takeoff weight in lower region of the plot, as indicated by the average line. It appears that there are several MTOW data points that are way higher than the majority others. This observation can be taken to imply that these points are outliers to the data set. Out of these 40 considered MTOW data points, the highest of them is recorded as $86,000 \mathrm{~kg}$ whereas the lowest one is only $930 \mathrm{~kg}$. This is such a big spread for the data values, with the average value is calculated to be $16,144.65 \mathrm{~kg}$.

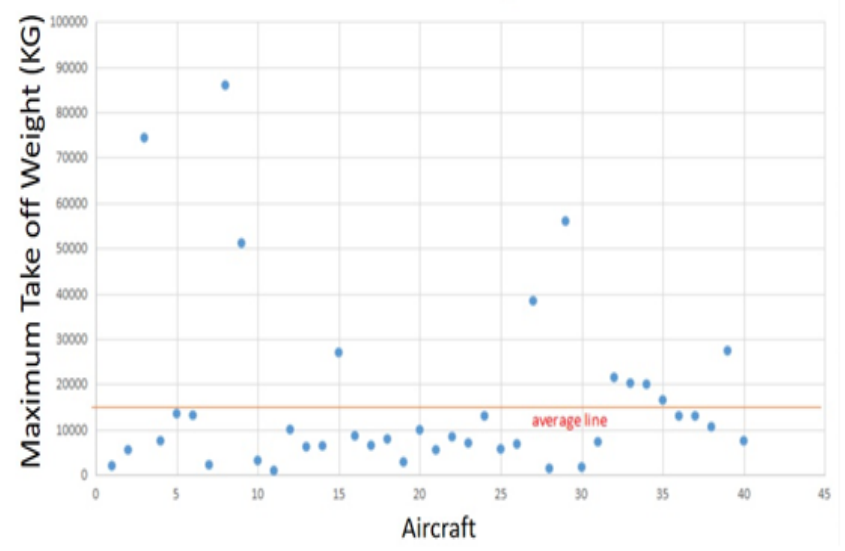

Fig.2. Maximum takeoff gross weight of the search and rescue aircraft

Using the graphical mean method, all of the outliers have been identified as illustrated in Fig. 3. These identified outlier data points are removed and the resultant scatter plot without them is presented in Fig. 4. It can be observed in Fig. 4 that the average MTOW value has now shifted down to $8,649.58$ $\mathrm{kg}$ as compared to the previous $16,144.65 \mathrm{~kg}$. The difference is rather significant, which indirectly highlights the effects of the outliers to the data analysis. In terms of the spread of the data points, they are still scattered but with more reasonable range of values.

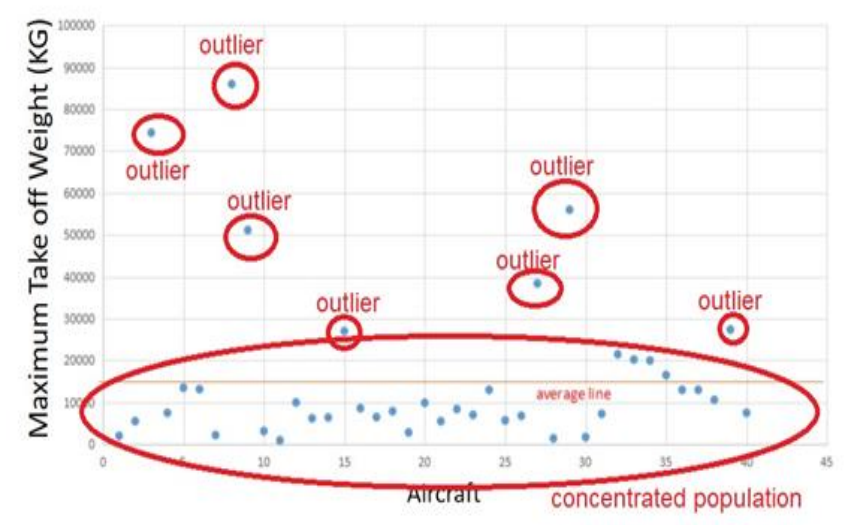

Fig.3. Outlier data identification 


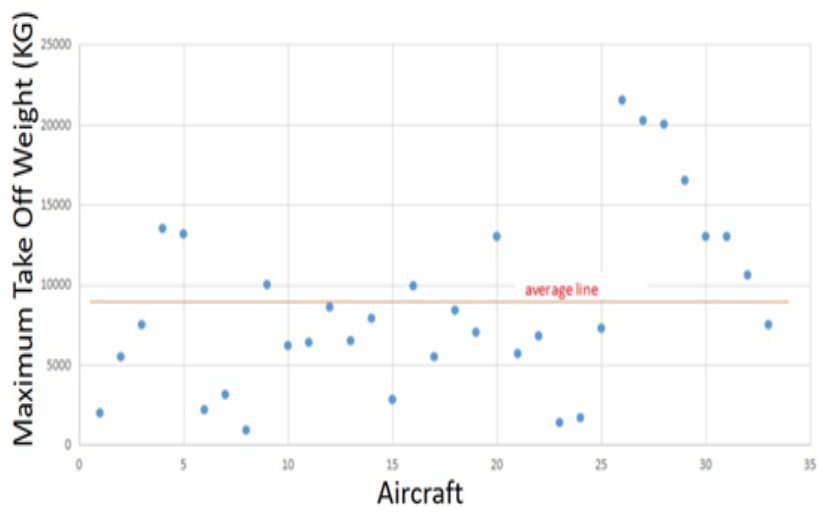

Fig.4. Maximum takeoff gross weight of the search and rescue aircraft without outliers

With removal of the seven outlier data points, the highest MTOW value for the improved data set is $21,500 \mathrm{~kg}$ while the lowest value is just $930 \mathrm{~kg}$. It should be noted that the highest MTOW value corresponds to Antonov An-140 aircraft, which is capable of accommodating up to maximum 50 passengers in the non-search and rescue design setting. In typical design modification for use in search and rescue mission, and taking into account the placement of medical equipment onboard, it is still capable to ferry between 20 to 30 people [12]. On the other end of the spectrum, the lowest MTOW value belongs to the Schweizer Model 300 aircraft, which is a helicopter that is only capable of accommodating one injured individual. This aircraft is primarily operated for reconnaissance sorties. The modified MTOW data set of considered aircraft that have been typically used in search and rescue sorties has been categorized into several groups according to their value. The data frequency for each of the groupings is tabulated and it is as presented in Table I. It can be observed from the tabulated data that the most of the aircraft fall within the $4,301 \mathrm{~kg}$ to the $8,600 \mathrm{~kg}$ of MTOW category, which captures $42.42 \%$ of the total considered aircraft in this study. This finding is perfectly in line with the average MTOW value that has been calculated from the modified data set without the outlier data points. By looking further into these aircraft designs, it can be concluded in a simplistic manner that most search and rescue aircraft has the capacity to accommodate six people on average. It must be considered that most of these aircraft have to be installed or equipped with medical equipment and therefore there will be some forfeited seats or capacity to hold more passengers [13].

Table-I: Data frequency of MTOW for aircraft commonly used in search and rescue sorties

\begin{tabular}{|c|c|}
\hline Range of MTOW (kg) & Frequency \\
\hline $17201-21500$ & 3 \\
\hline $12901-17200$ & 6 \\
\hline $8601-12900$ & 3 \\
\hline $4301-8600$ & 14 \\
\hline $0-4300$ & 7 \\
\hline
\end{tabular}

Overall, 40 aircraft that have been operated for search and rescue missions are identified and also analyzed. Based upon the scatter plot (without outliers) of MTOW, it seems that on average, most operators are utilizing medium haul aircraft that can carry loads or injured people within the range of MTOW between 4,301 kg and 8,600 kg for search and rescue sorties. It has also been projected that most of these aircraft can carry up to six passengers on average. With this knowledge, it can be referenced when designing new aircraft for the search and rescue missions, or when selecting the appropriate aircraft for such operations.

\section{REFERENCES}

1. AW149 Multi-Mission Performance [Online]. Available: www.leonardo company.com/en/products/aw149

2. Dornier 228 [Online]. Available: https://dornier228.ruag.com/en

3. J. Groves, A Combat Search and Rescue (CSAR) Role for the CV-22: It's Coming, Get Ready, Quantico, VA: Marine Corps University, 2008

4. V-22 Osprey [online]. Available: www.boeing.com/defense/v-22-osprey

5. S-70i Black Hawk Helicopter [Online]. Available: https://www.armytechnology.com/projects/s-70i-black-hawk-helicopter/

6. J. Laurikkala, M. Juhola, and E. Kentana, "Informal identification of outliers in medical data," International Workshop on Intelligent Data Analysis in Medicine and Pharmacology, Berlin, Germany, 2000

7. G. H. John, "Robust decision trees: Removing outliers from database," International Conference on Knowledge Discovery and Data Mining, Montreal, Canada, 1995

8. E. Acuna, and C. Rodriguez, "On detection of outliers and their effect in supervised classification," International Partnership for the Satoyama Initiative (IPSI) Conference, Venice, Italy, 2004

9. S. Pandey, S. Muthuraman, and A. Shrivastava, "Data classification using machine learning approach," International Symposium on Intelligent Systems Technologies and Applications, Bangalore, India, 2018

10. Boldon James, What is Data Classification? [Online]. Available: www. boldonjames.com/what-is-data-classification-definition/

11. Identifying Outliers [Online]. Available: https://support.minitab.com/en -us/minitab/18/help-and-how-to/statistics/basic-statistics/supporting-to pics/data-concepts/identifying-outliers/

12. Antonov An-140 Regional Passenger Airliner [Online]. Available: www.aerospace-technology.com/projects/antonov-an-140/

13. $M H-139$ [Online]. Available: www.boeing.com/defense/mh-139/index. page

\section{AUTHORS PROFILE}

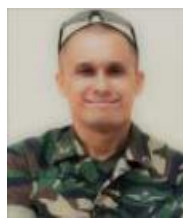

Mohd Harridon Mohamed Suffian has been involved in a number of search and rescue missions and he holds several certifications in Search and Rescue, and Physical Fitness. He is affiliated with few organizations including the National Association for Search and Rescue of USA, Flight Test Society of Australia, Universiti Kuala Lumpur MIAT, European Paratroopers, and others. He is also the President of St John Ambulance Putrajaya.

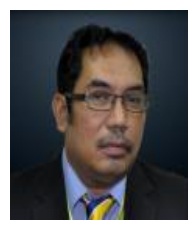

Mohamad Dali Isa is a Professor and Dean at Universiti Kuala Lumpur MIAT. He has extensive experiences in the aviation industry and is central figure in aircraft structure. He obtained his education in the United Kingdom and Australia, and was a key member in Technical Services Department of Malaysia Airlines. He authored numerous academic and technical papers and is also a sought after keynote speaker. Due to his contributions, he was awarded the Datukship by the Sultan of Pahang. 
Hazariah Mohd Noh is the Deputy Dean at Universiti Kuala Lumpur MIAT and is expert in aviation biofuels. She obtained her education in USA and Spain, and gained valued experience in aircraft maintenance from Malaysia Airlines and several other organizations. She obtained the MARA Scholarship Award for Best Student in 2003. In addition, she also oversees several postgraduate students and was attached to Airbus Military in Madrid in 2009.

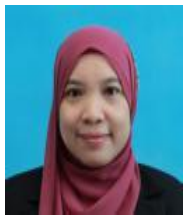

Nurhayati Mohd Nur is the current Head of Research and Innovation at Universiti Kuala Lumpur MIAT. Her expertise is in the field of quality assurance where she had applied her expertise in both automotive and aerospace industries. She has several postgraduate students under her doing research in aerospace engineering field. She is currently collaborating with the Malaysian Military with regards to ergonomics of aircraft. 